\title{
Simulation studies of polymer translocation through a channel
}

\author{
H. C. Loebl, R. Randel, S. P. Goodwin, and C. C. Matthai \\ Department of Physics and Astronomy, University of Wales, Cardiff, United Kingdom
}

(Received 11 September 2002; revised manuscript received 3 December 2002; published 29 April 2003)

\begin{abstract}
Monte Carlo simulation studies of the translocation of homopolymers of length $N$ driven through a channel have been performed. We find that the translocation time $\tau$ depends on temperature in a nontrivial way. For temperatures below some critical temperature $\theta_{c}, \tau \sim T^{-1.4}$, whereas for $T>\theta_{c}, \tau$ increases with temperature. The low temperature results are in good agreement with experimental findings as is the dependence of $\tau$ on the driving field strength. The velocity of translocation displays the same characteristics as found in experiment but the $N$ dependence of $\tau$ shows the linear relationship observed in experiment only for large values of $N$. A possible reason for this is suggested.
\end{abstract}

DOI: 10.1103/PhysRevE.67.041913

PACS number(s): 87.14.Gg, 87.15.He, 87.15.Aa

\section{INTRODUCTION}

In recent years there has been much interest in the study of the translocation of biopolymers through channels under the influence of external fields. For example, Kasianowicz et al. [1] showed that an electric field can be used to drive single stranded RNA or DNA molecules through a narrow ion channel in a lipid bilayer membrane. They found that the channel blockade lifetime was proportional to the mean polymer length and inversely proportional to the applied voltage. Meller et al. [2] extended these investigations to study the temperature dependence of the translocation duration. Subsequent investigations [3] found that the velocity of the biopolymers in the channel depended on the relative size of the polymer compared to the pore length.

In parallel with these experimental investigations, there have been numerous theoretical and computational studies of polymer translocation. These have, in the main, been restricted to considering the one-dimensional (1D) driftdiffusion model [4-6]. Chuang et al. [7] performed numerical simulations and showed that translocation times of selfavoiding two-dimensional chains scale with the size of the chain, $N$, in the same way as the diffusion times. They predicted the average pulling velocity of the polymer to depend on $N$. Chern et al. [8] performed 3D Monte Carlo (MC) simulations of a polymer translocating through a hole in a planar slab. However, in this case the slab thickness was taken to be the intermonomer separation and so may not be directly comparable with the experimental studies referred to above in which the channel was of the order of a few intermonomer separations. The related problem of the translocation of a confined polymer through a hole was considered by Muthukumar [9].

We have endeavored to construct a model in order to perform simulations of polymer translocation, which may be compared with available experimental data. The model and the simulation techniques are outlined in the following section. This is followed by a presentation of the results showing how the translocation times of a polymer depend on the temperature at which the simulation is run and on the strength of the external field. The dependence of the translocation times and velocity on the length of the polymer is also addressed. Finally, the results are discussed with reference to other simulation studies and experimental observations.

\section{MODEL AND SIMULATION TECHNIQUES}

In trying to model the translocation of a DNA (or RNA) molecule through a $\alpha$-hemolysin pore in a membrane, a minimalist approach was taken. The molecule, of contour length $L=N a$, was treated as a homopolymer comprising $N$ beads, each representing a base, with nearest neighbor bond length, $a$. Two potential forms, the Lennard-Jones and Morse, were used to describe the intermonomer interactions. Both gave similar results for the distributions of translocation times, but the distributions at low temperatures with the Morse potential resulted in better defined peaks. Since this is necessary to extract reliable peak translocation times, the results given in this paper are those obtained using the Morse potential

$$
\begin{gathered}
V_{i j}=\epsilon\left\{\exp \left[-2 \alpha\left(r_{i j}-a\right)\right]-2 \exp \left[-\alpha\left(r_{i j}-a\right)\right]\right\}, \quad r_{i j}<r_{c}, \\
V_{i j}=0, \quad r_{i j}>r_{c},
\end{gathered}
$$

where $r_{i j}$ is the distance between two monomers $i$ and $j$. All the distances in the simulation were taken in units of the bead radius $\sigma$ defined through the condition $V_{M}(\sigma)=0$. Similarly, taking $\epsilon$ to be unity establishes the unit of energy and the temperature of the simulation is then given in units of $\epsilon / k_{B}$. By taking the cutoff radius $r_{c}$ to be $1.5 \sigma$ and the parameter $\alpha$ to be $10 / \sigma$, this potential allows for the modeling of the different conformations of polymers as the temperature is varied, and in particular, gives rise to a globuleextended chain transition at the $\theta$ temperature [10].

The bond energy associated with adjacent monomers $i$ and $i+1$ was represented by the potential

$$
V_{b}=-0.5 \gamma R_{\text {max }}^{2} \ln \left[1-\left(r_{i, i+1} / R_{\text {max }}\right)^{2}\right], \quad r_{i, i+1}<R_{\text {max }} .
$$

The maximum bond length over which this interaction is nonzero was taken to be the same as for the Morse potential; $R_{\text {max }}=1.5 \sigma$ and taking the constant $\gamma$ to be $30 \epsilon / \sigma^{2}$ results in a fairly rigid bond. 
The $\alpha$-hemolysin pore through the membrane was represented by a cylindrical tube of length $W=12 a$ and width small enough $(a)$ so as to ensure that the polymer was stretched out in the tube. This discounts the possibility of hairpin translocations that are not expected to take place. The membrane itself was set up as an impenetrable wall (Fig. 1). The dimensions of the pore are consistent with the self-assembled nanopore of Meller et al. [3].

The external driving force was modeled as a potential difference applied linearly across the length of the pore with the profile in the manner described by Chern et al. [8],

$$
V_{m e m}= \begin{cases}-\kappa x, & x \geqslant-W / 2 \text { and } x \leqslant W / 2 \text { and } y^{2}+z^{2} \leqslant R^{2} \\ -\kappa W / 2, & x>W / 2 \\ \kappa W / 2, & x<-W / 2 \\ \infty & \text { otherwise, }\end{cases}
$$

where $R$ is the radius of the pore and $\kappa$ is the strength of potential.

To begin the simulation of the translocation of the polymer through the pore, from left to right as shown in Fig. 2, a chain is placed on one side of the pore with one end of it in the pore. This is accomplished by constructing the polymer chain as follows. The first monomer is initially positioned at the pore entrance and the positions of the monomers comprising the rest of the chain obtained from a self-avoiding random walk on a cubic lattice of spacing $a$. The walk is constrained so that it does not cross the membrane wall. Thus, at the start, the whole chain is to the left of the pore. The chain is then allowed to reach an equilibrium conformation using the standard Monte Carlo method, but with the constraint that the first monomer, placed at the pore edge, is fixed. The move set employed is to pick a monomer at random and then allow it to make a trial move of $0.05 \sigma$ in either $\pm x, \pm y$, or $\pm z$ direction. The probability of accepting a move taking a monomer from one state to another is given by the Metropolis criterion.

Once the polymer is in its equilibrium configuration, the first monomer at the pore entrance is released from its constraint and the simulation of the polymer moving through the pore begins. For a set of starting parameters, the length of time taken for the molecule to move through the pore is the
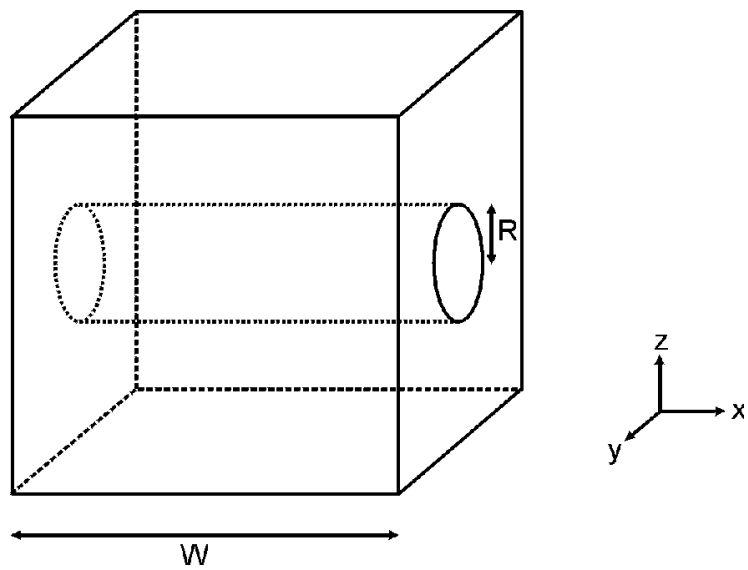

FIG. 1. Schematic illustration of the membrane with the pore. translocation time. Not all simulations result in a successful translocation and even when they do, the times vary over a wide range. The success rate, defined as the fraction of successful runs, depends on the temperature at which the simulation is run. At high temperatures, the chain entropy is high and when it acts against the potential difference across the membrane, the chain drops out of the tube. This does of course depend on the fraction of the chain that is outside the tube. At low temperatures, the pulling force is not strong enough to uncoil the globulelike polymer making transloca-

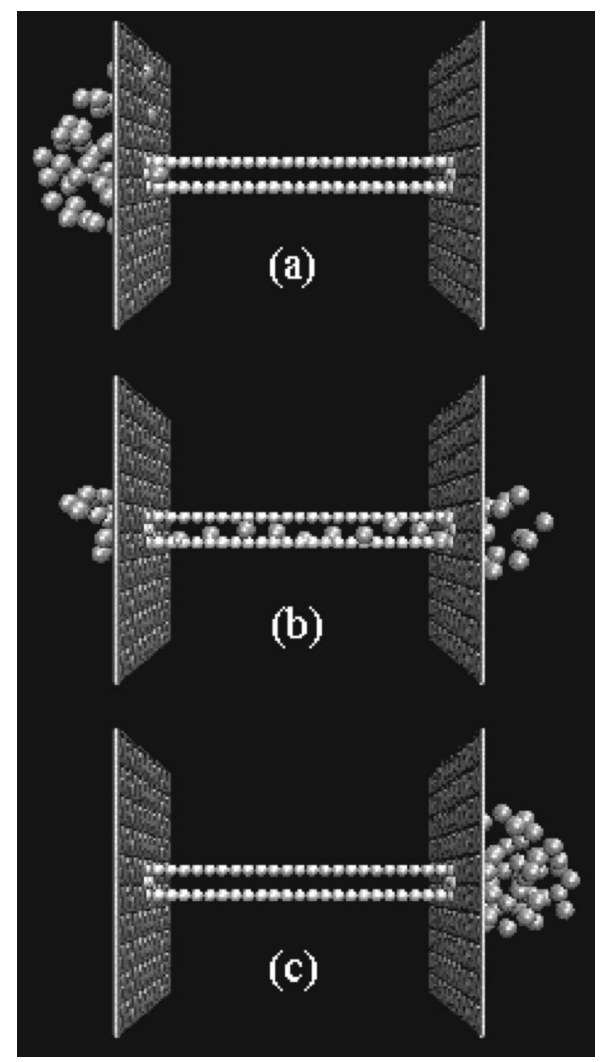

FIG. 2. Snapshots of the polymer (a) at the start of a translocation run, (b) during the simulation, and (c) once the translocation has been completed. The pictures were created using the visual molecular dynamics package [11]. 
tion impossible. Thus, at high and low temperatures, the success rate is much less than unity. In order to have a consistent way of determining the translocation time at each temperature, we have followed the prescription of Meller et al. [3], hereafter referred to as MNB, and taken $\tau$ to be the most probable translocation time from a set of at least 500 simulations.

It is not easy to relate a MC step to a duration in real time. However, we can consider each accepted move, a move that satisfies the Metropolis criterion, to be equivalent to a fraction of a vibration of the monomer. Then $\tau=t_{0} n_{A}$, where $t_{0}$ is the time taken for a monomer to move the $0.05 \sigma$ associated with an accepted move and $n_{A}$ the number of steps taken for the translocation to be completed. We estimate that, taking an average monomer mass to be $100 \mathrm{amu}$, at room temperature this is of the order of $0.1 \mathrm{ps}$. As the number of steps required for a typical translocation is of the order of $10^{7}$, this results in translocation times of the order of microseconds. This is of course much too fast when compared with experiment, but it must be noted that our simulations do not take into account any drag or polymer-wall interactions. Such effects will, in general, have the effect of increasing the factor $t_{0}$ manyfold. In the presentation of our results, we have taken $t_{0}=10^{-10} \mathrm{~s}$. This gives translocation times of the order observed in the experiments making comparisons easier.

\section{RESULTS OF SIMULATIONS}

\section{A. Variation of $\tau$ with temperature}

To determine how $\tau$ depends on the temperature, we considered a polymer chain of length $N=30$ which is two and a half times the length of the pore. The strength of the driving potential was taken to be equal to $5 \epsilon$. It turns out to be the optimum strength, being the smallest field which gives fairly fast translocation times thereby making the studies feasible. The simulations were performed for a range of temperatures $0.2 \leqslant k_{B} T / \epsilon \leqslant 1.0$ and at each temperature at least 500 simulation runs were carried out and the translocation time distribution plotted as a histogram [Fig. 3(a)]. The variation of the most probable translocation times $\tau$ (the peaks in the histograms) with temperature is shown in Fig. 3(b). It can be seen that there are two distinct regions. For $T$ greater than some critical temperature $\theta_{c}$ (the temperature corresponding to the minimum in $\tau$ ), $\tau$ increases with $T$. By contrast when $T$ $<\theta_{c}, \tau$ decreases with increasing $T$. In this latter region, the variation is well fitted by an inverse power law relationship

$$
\tau \sim T^{-1.36}, \quad T<\theta_{c} .
$$

In order to understand these observations, one notes that there are two components that present a barrier to the pulling force arising from the potential difference across the membrane. The first $E_{c}$ is due to the intermonomer potential of Eq. (1), which makes it energetically favorable for the polymer to adopt compact conformations. If the polymer is in such a conformation, the pulling force has to first unwind the polymer by breaking the intermonomer interactions before dragging it through the pore. The other arises from the tem-
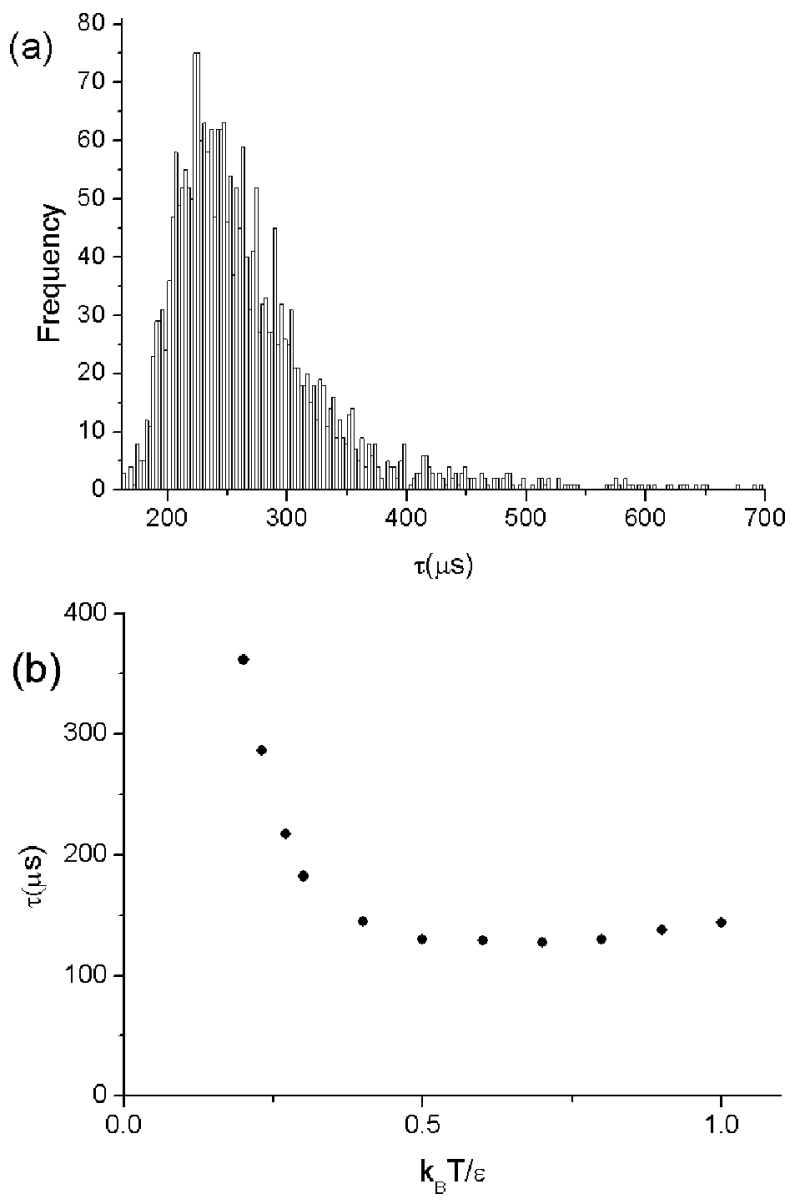

FIG. 3. The (a) distribution of translocation times, (b) the variation of the most probable translocation time $\tau$ with temperature, for a polymer of length $N=30$ with an applied force constant $\kappa / \epsilon$ $=5$.

perature fluctuations and is an entropy barrier $E_{e}$. Whereas $E_{e}$ increases with temperature, the cohesive energy barrier $E_{c}$ will reduce as $T$ is increased. The consequence of this is that the net barrier to pulling displays a minimum at the critical temperature $\theta_{c}$ (Fig. 4).

In their study of DNA polymers electrophoretically driven through a nanopore, Meller et al. [2] found a $T^{-2}$ dependence of the translocation process. The fact that we find a much less strong temperature dependence is a reflection of the simplistic way in which the drag was incorporated. However, it is clear that the low temperature variation found in our simulations is a direct consequence of the intermonomer interactions. A model of the type sketched above would also explain the striking differences observed between the different polymers in the experimental study [2].

\section{B. The effect of varying the field strength}

Having established the effect of temperature on the translocation process, we proceeded to determine the influence of the field strength on $\tau$. Kasianowicz, Brandin, Branton, and Deamer (KBBD) [1] found that the channel blockade lifetimes were inversely proportional to the applied voltage. By contrast, the studies of MNB on the polymer velocities dur- 


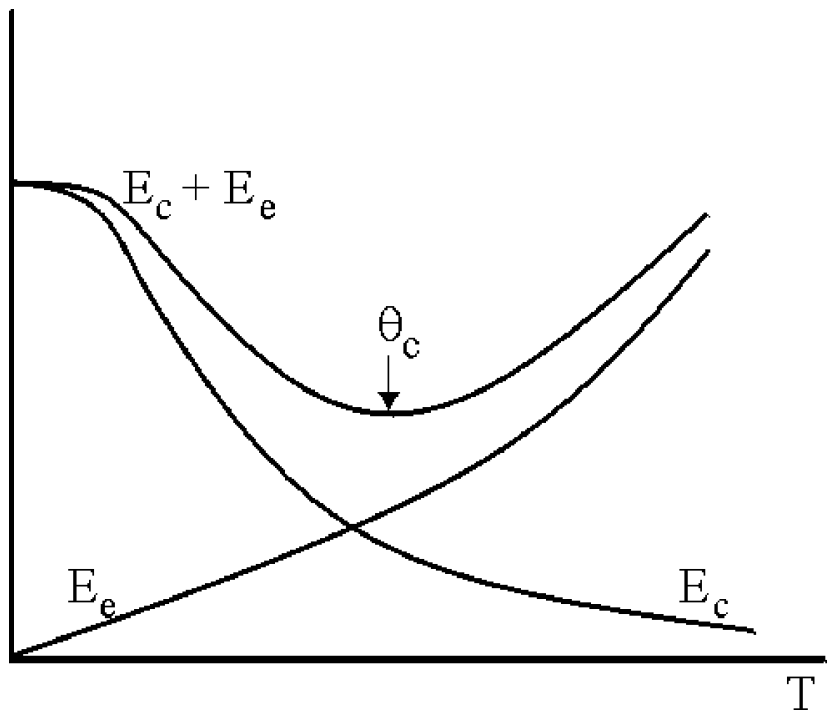

FIG. 4. Schematic showing the variation of the cohesive energy and entropy barriers resulting in a minimum at $\theta_{c}$.

ing translocation suggest a quadratic dependence on the applied field. If there is a barrier to translocation, in the manner suggested above, there should be a minimum field strength below which no translocation is possible. We carried out a series of simulations for a fixed polymer length $(N=30)$ and temperature $k_{B} T / \epsilon=0.3$, for a range of values of the force constant, $1<\kappa / \epsilon<10$. The translocation times thus obtained are shown in Fig. 5 . For the small values of $\kappa$, there are very few successful runs and those that do make it through the pore tend to take a very long time. For large values of $\kappa$, the times do not vary much which is an indication that the field strength is so strong that the barrier effects are not seen. The results point to an inverse power dependence of the form $\tau$ $\sim\left(\kappa-\kappa_{0}\right)^{-\nu}$ and we did indeed find a reasonably good fit to the data with $\kappa_{0}=0.15 \epsilon$ and $\nu=1.0$. The inverse dependence on the applied field is in good agreement with the results of KBBD. It may be noted that the value of $\nu$ appears to depend on the intermonomer potential used. For example, using a different (Lennard-Jones) intermonomer potential we found that the field dependence of the translocation time was well fitted by an inverse quadratic form. However, both these sets

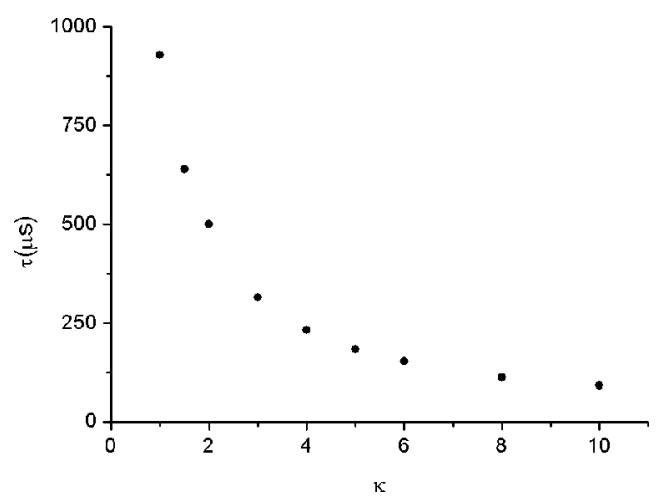

FIG. 5. The variation of $\tau$ with the field strength $\kappa$ (in units of $\boldsymbol{\epsilon})$ for a polymer of length $N=30(\bullet)$ at temperature $k_{B} T / \epsilon$ $=0.3$.

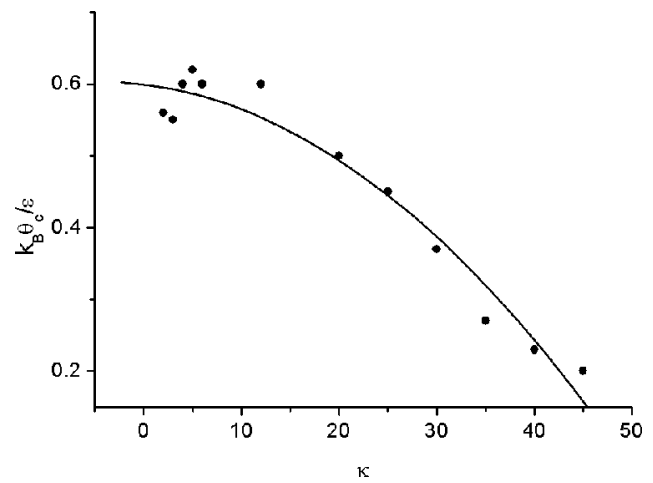

FIG. 6. The dependence of the critical temperature $\theta_{c}(\mathbf{O})$ on the force constant $\kappa$ (in units of $\epsilon$ ) is well fitted by a quadratic form (line).

of results point to a inverse power law dependence with $\nu$ somewhere in the range 1-2. This is in broad agreement with both KBBD and MNB. Assuming that these simulations correspond to room temperature results, we estimate the threshold $\kappa_{0} \sim 13 \mathrm{mV}$. Although this is a bit lower than that estimated by MNB, the agreement between the two is encouraging especially given the simplistic form of the potential used.

To further test our hypothesis of the existence of a barrier of the type speculated above, we performed simulations to determine the dependence of $\theta_{c}$ on the field strength. Since increasing the field strength would have the property of reducing the effective $E_{c}$ barrier but no effect on $E_{e}, \theta_{c}$ should correspondingly decrease. This is indeed what we observed in our simulations, the results of which are shown in Fig 6.

\section{Dependence of the translocation on polymer length}

In order to determine the $N$ dependence of the translocation times and velocities, we performed a series of simulations on polymers of different lengths at temperature $k_{B} T / \epsilon$ $=0.3$. The choice of temperature was influenced by the $\tau(T)$ results in Fig. 3 which show clearly that the experimental data, with which our results are compared, relate to the low temperature region of our simulations. The resulting translo-

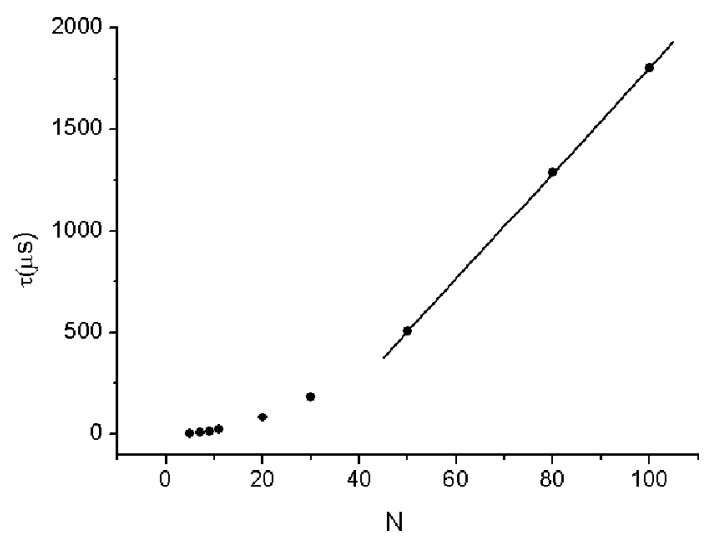

FIG. 7. The variation of the translocation times with the number of monomers, $N$, for $\kappa / \epsilon=5$ and $k_{B} T / \epsilon=0.3$. For $N \geqslant 50$, the data are well fitted by a straight line. 


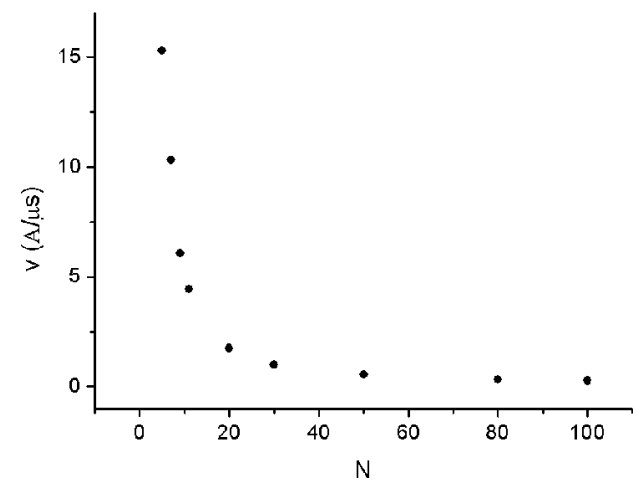

FIG. 8. The variation of velocity $v$ with the number of monomers, $N$, for field strength $\kappa / \epsilon=5$ and $k_{B} T / \epsilon=0.3$.

cation time dependence on $N$ (shown in Fig. 7) is clearly nonlinear for $N$ smaller than the pipe length. For larger polymers, however, $\tau$ appears to increase linearly with $N$ which is in agreement with the experimental observations [1,3]. The average polymer velocity was determined by simply dividing the length of the pore plus the polymer length by the translocation time, $v=(L+W) / \tau$. For polymers longer than the pore length, the velocity plateaus out showing its independence on $N$ (Fig. 8). The plateau value itself is dependent on the temperature at which the simulations are run. At $k_{B} T / \epsilon$ $=0.3$, the velocity for large $(N \geqslant 50)$ polymers plateaus at $0.3 \AA / \mu$ s. For the shorter polymers (below the pore length), the velocity is found to increase nonlinearly for decreasing polymer lengths. Although both these features are in agreement with the findings of MNB [3], the very high velocities of short polymer chains are not. In order to understand this discrepancy, we made a detailed examination of the translocation process. We found that the short polymers appeared to keep to a very rigid conformation whilst moving through the pore in contrast with the longer chains that executed undulations during their progress through the pore. This difference in behavior may be attributed to the polymer segment outside the pore. Fluctuations and vibrations of the monomers in the outside segments are transmitted to those monomers inside the pore making it relatively slower.

\section{DISCUSSION}

We have shown that a minimalist model of polymers can be useful in simulating their translocation through pores. To obtain translocation times that are of the order measured in experiments, it is necessary to take into account the drag and polymer-pore interactions. However, these may be taken into account in a simplistic fashion by introducing a drag factor. Although we have taken this factor to be independent of $N$ and $T$, it would appear that the results are to some extent meaningful and directly comparable with experiment. We have been able to show that the translocation time dependendence on temperature arises from the entropy barrier of the polymer having to squeeze into the pore and from intrapolymer interaction which resists the unwinding necessary for it to travel through the pore. This was borne out by our results on the field strength dependence, which is in general agreement with experiment. We have also established that the experiments would correspond to the low temperature simulations. The velocity dependence on $N$ was also found to be in agreement with experiment, but only for large $N$. The discrepancies between experiment and the results of these simulations may be attributed to two shortcomings in our model, viz., the simple intermonomer potential used and the assumption that the drag is the same at all temperatures. We are currently investigating this by performing Langevin molecular dynamics simulations in which the drag is not the same at all temperatures. Preliminary results suggest a temperature dependence in better agreement with experiment [12].

\section{ACKNOWLEDGMENTS}

H.C.L. and R.R. acknowledge financial support from the University of Wales. We thank Dr. R. Philp for useful discussions and for helping us in carrying out the simulations on the CSPACE parallel computer facility at Cardiff.
[1] J.J. Kasianowicz, E. Brandin, D. Branton, and D.W. Deamer Proc. Natl. Acad. Sci. U.S.A. 93, 13770 (1996).

[2] A. Meller, L. Nivon, E. Brandin, J. Golovchenko, and D. Branton, Proc. Natl. Acad. Sci. U.S.A. 97, 1079 (2000).

[3] A. Meller, L. Nivon, and D. Branton, Phys. Rev. Lett. 86, 3435 (2001).

[4] W. Sung and P.J. Park, Phys. Rev. Lett. 77, 783 (1996).

[5] M. Muthukumar, J. Chem. Phys. 111, 10371 (1999).

[6] D.K. Lubensky and D.R. Nelson, Biophys. J. 77, 1824 (1999).
[7] J. Chuang, Y. Kantor, and M. Kardar, Phys. Rev. E 65, 11802 (2001).

[8] S.S. Chern, A.E. Cardenas, and R.D. Coalson, J. Chem. Phys. 115, 7772 (2001).

[9] M. Muthukumar, Phys. Rev. Lett. 86, 3188 (2001).

[10] R.G. Maurice and C.C. Matthai, Phys. Rev. E 60, 3165 (1999).

[11] W. Humphrey, A. Dalke, and K. Schulten, J. Mol. Graphics 14, 33 (1996).

[12] R. Randel, H. C. Loebl, and C. C. Matthai (unpublished). 